\title{
Sensitization Mechanisms of Photopolymer Coating Layer using Infrared Dye
}

\author{
Takeshi Hatano ${ }^{\text {n) }}$, Keijiro Fukui ${ }^{\text {a) }}$, Takashi Karatsua ${ }^{\text {a) }}$, Akihide Kitamura ${ }^{\text {a) }}$ and
} Toshiyuki Urano*b)c

a) Department of Materials Technology, Faculty of Engineering, Chiba University, Inage-ku, Chiba 263-8522, Japan.

b) Center for Cooperative Research, Chiba University, Inage-ku, Chiba 263-8522, Japan

c) Research Center, Mitsubishi Kagaku Corporation, Aoba-ku, Yokohama 227 Japand

Photoinitiation mechanisms of photopolymer coating layer containing a heptamethine cyanine dye and a radical generating reagent were investigated by laser flash photolysis using a total reflection cell. From fluorescence quenching studies, it was found that singlet static sensitization process was predominant for CDB-TCT systems.

Keyword: infrared photoinitiator, photopolymer, sensitization, infrared dye.

\section{Introduction}

The photoinitiation mechanism for photopolymer coating layer containing a heptamethine cyanine dye $2-[2-[2-$ chloro- $3[2-$ (1,3-dihydro-3,3-dimethyl-1-methoxyethyl-2Hindol-2-ylidene)-ethylidene]-1-cyclohexene-1yl]-ethenyl]-3,3-dimethyl-1-methoxyethyl-1Hindolium tetrafluoroborate (CDB) and a radical generating reagent 2,4,6-tris (trichloromethyl)1,3,5-triazine (TCT) was investigated by laser flash photolysis using a total reflection cell as depicted in Fig. 1.

It is well known that infrared cyanine dyes are used for fluorescenct probes in biochemical procedure[1], sensitizer dyes of silver halide photography[2], dyes for dye laser technology [3], and abration dyes for write-once-opticalmenory discs.
The character of photopolymer sensitization of cyanine dyes was not reported except for some reports $[4,5]$. In our earlier investiagtion, [4] $20 \mathrm{mJcm}^{-2}$ of sensitivity was obtained at $830 \mathrm{~nm}$, and the sensitization mechanisms are not clear yet.

According to the rapid development of highpower-laser diodes, the infrared-light-responsible photopolymers are demanded for new imaging systems equipped with laser diode with compact side, low price, and long shelf life compared to visible laser imaging systems.

Sanchez-Galvez et al. described the calculation of potential energy surface for visible and infrared cyanine dyes, and discussed an ultrafast radiationless deactivation process of the dyes by comparing results of calculation with that of photolysis in solution[5]. The ultrafast

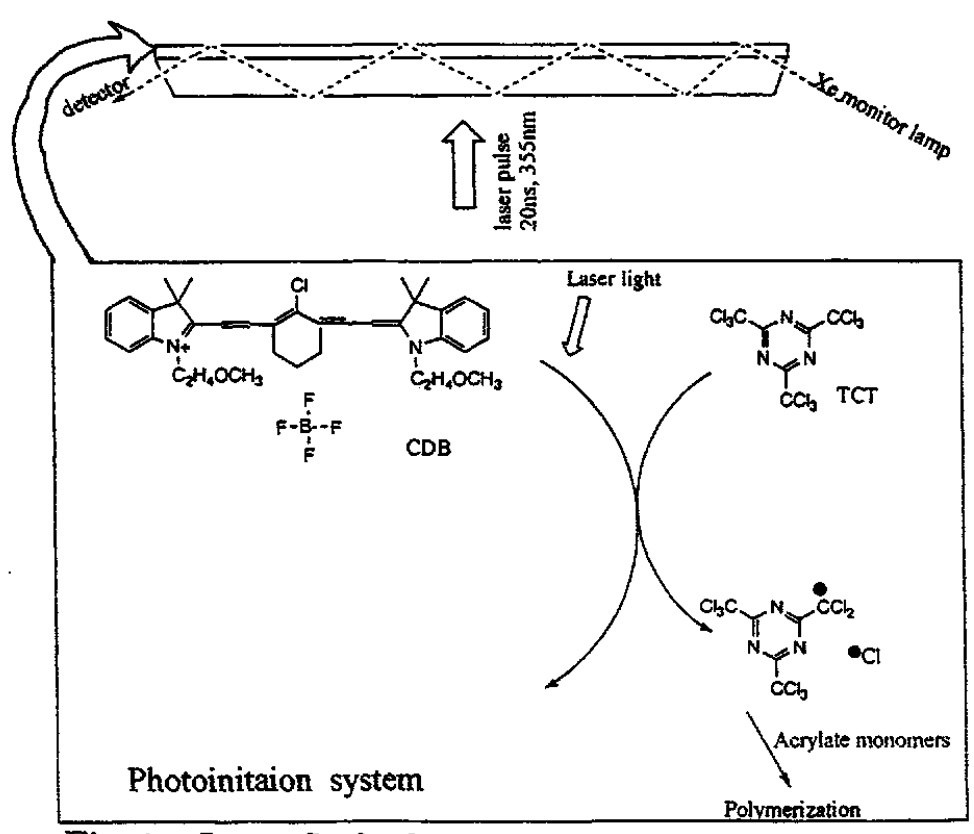

Fig. 1. Laser flash photolysis. 

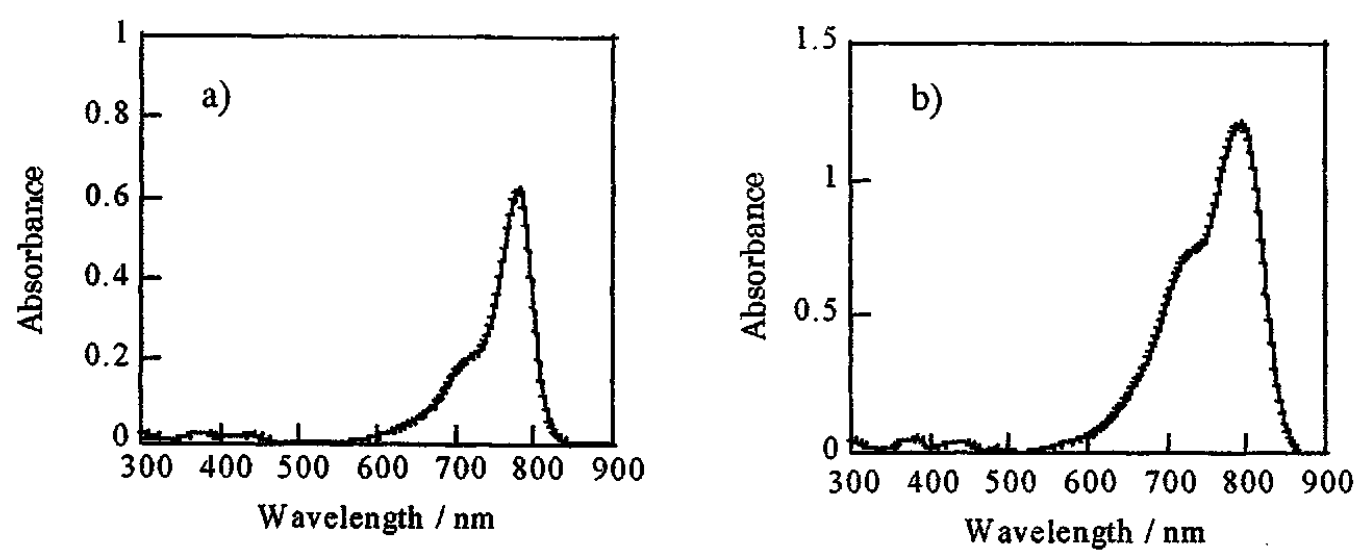

Fig. 2. Absoption spectrum of CDB in acctonitrile a), and in PMMA b).

Substance concentration: $3.1 \times 10^{-6}$ and $1 \times 10^{-1} \mathrm{~mol} \mathrm{dm}^{-3}$ for $\mathrm{CDB}$ in acetonitrile and in PMMA.

radiationless deactivation process was a relaxation process through twisted intramolecular charge transfer (TICT) state from photoexcited singlet state accompanied with a rotation of polymethyne group, and the fast process made the decrease in fluorescence emmision, which resulted in a low-fluorescence quantum yield $\left(\Phi_{f}\right)$ for cyanine dyes such as $\Phi_{\mathrm{f}}=0.06-0.4[5]$, The low efficiency of fluorescence and low sensitivity of photodetector in infrared light region such as photomultipliers make it diffucult to analyse the fluorescence using a normal fluorescence spectrometer.

A multi-reflection cell in the laser flash photolysis works as a collector of fluorescence lights. The fluorescence lights emmitted from the photoexcited dyes in photopolymer coating layer are multi-reflected in the cell, efficiently directing to both sides of the cell. The fluorescence passing through the cell was easily measured by the detector equipped with an optical fiber head, contacted to the sidewall of the cell.

In this paper we present the sensitization processes using an infrared cyanine dye of similar concentration to comercial products in the photopolymer systems.

\section{Experimental}

CDB was purchased from Nippon Kayaku. 2,4,6-Tris(trichloromethyl)-1,3,5-triazine (TCT) was purchased from Midori Chemical. Poly(methylmethacrylate) (PMMA, $\mathrm{Mw}=$ 40000 ), was estimated by GPC (RI). All solvents were spectrograde and purchased from Wako Pure Chemical Industries.

UV-NIR spectra were recorded with a

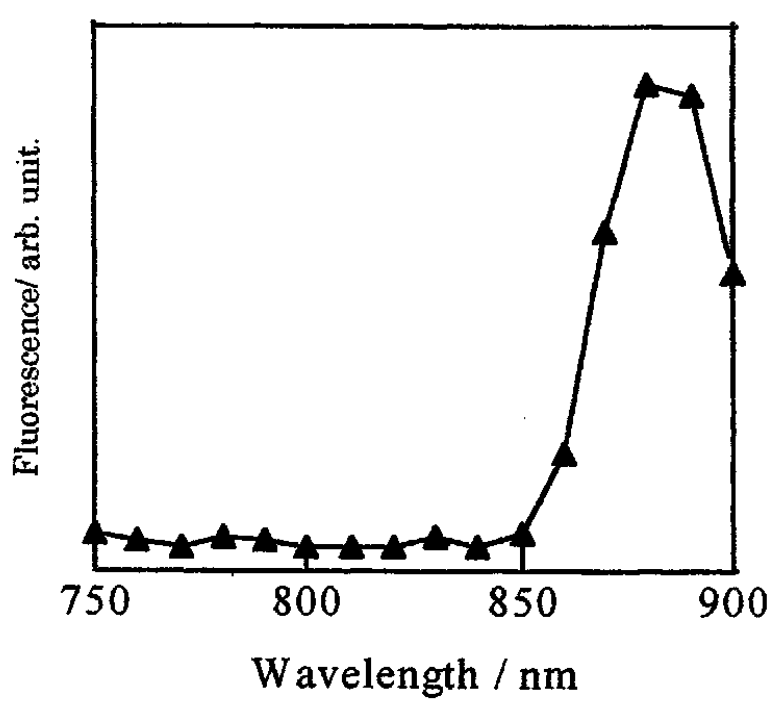

Fig. 3. Fluorescence spectra of $C D B$ in PMMA.

Substance concentration: $1 \times 10^{-1} \mathrm{~mol} \mathrm{dm}^{-3}$ for CDB; excitation wavelength $355 \mathrm{~nm}$.

Hiatchi U-3000 spectrophotometer. Laser flash photolysis in film was performed using a total reflection sapphire cell $(10 \times 30 \mathrm{~mm}, 1 \mathrm{~mm}$ thickness, and both short sides were cut at a 45 degree angle.). Films were spin-coated to form photopolymer layer as depicted in Fig. 1. Details concerning the apparatus are described elsewhere. $[6,7]$ An excitation light pulse (20 ns, $355 \mathrm{~nm}$ and $10 \mathrm{~mJ} / \mathrm{cm}^{2}$ per pulse) from a YAG laser (Spectron Laser System Model SL 402) was exposed over the entire sample cell. A monitoring light from a xenon lamp was passed through the multireflection cell which was connected to the head of an optical fiber attached to a monochromator (Instruments Digikrom 240) 


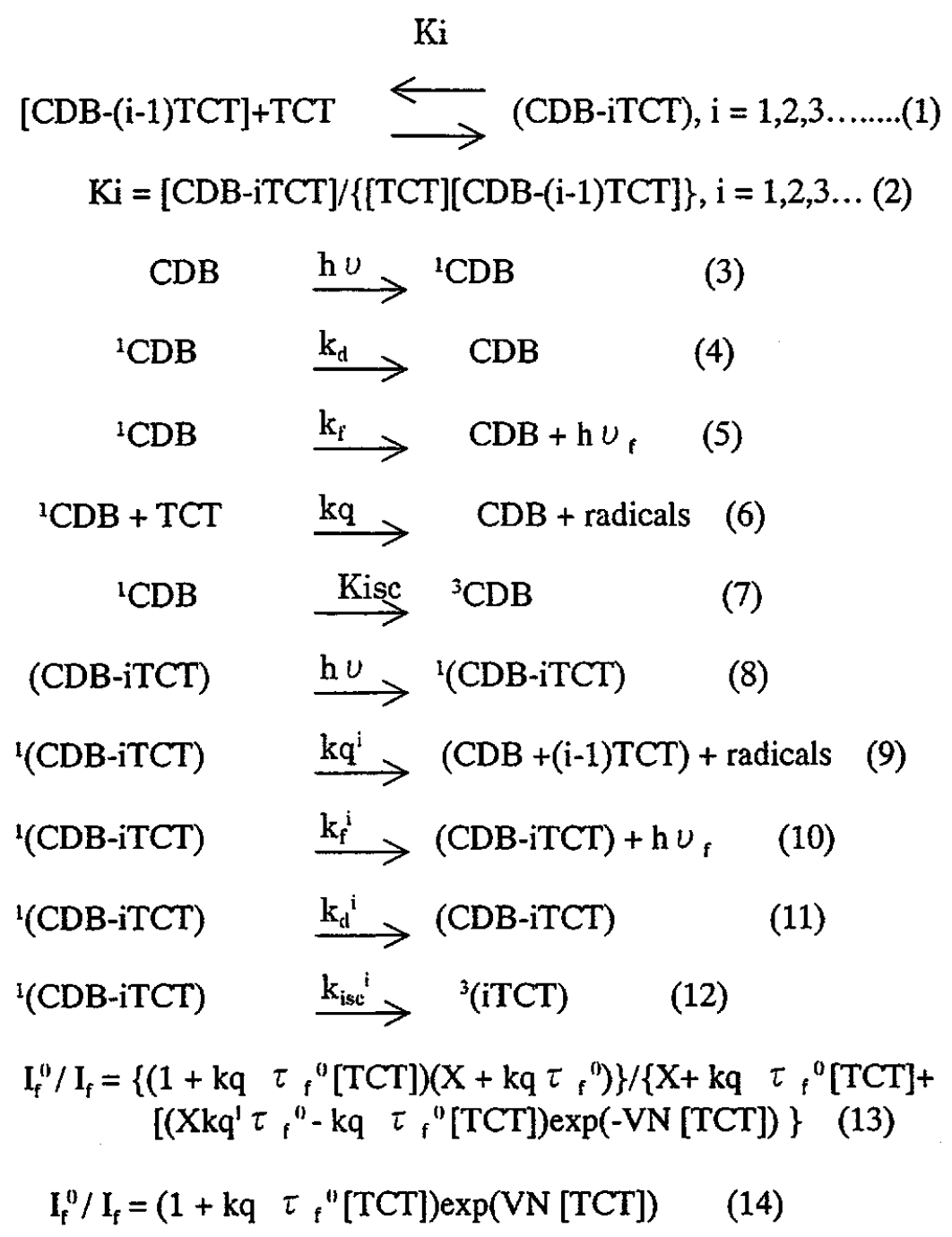

Where $I_{f}^{b}$ and $I_{f}$ are the fluorescence intensities in the absence and presence of the quencher (TCT), $\tau_{i}{ }^{\prime}$ is the life time in the absence of the quencher, $\mathrm{kq}$ is the dynamic quenching rate conastant, $\quad \mathrm{kq}^{l}$ is the static quenchng constant and $\mathrm{kq}^{\prime}=\mathrm{ikq}, \quad \mathrm{K}_{\mathrm{l}}=\mathrm{i} \mathrm{K}_{\mathrm{i}}=\mathrm{VN} . \quad \mathrm{V}$ is the quenching shere, $V=4 \pi R_{f}^{3} / 3$. N is Avogadro's number. $R_{f}$ is the quenching radius. $X=K_{1} \Sigma$ $[(\mathrm{CDB}-(\mathrm{i}-1) \mathrm{TCT})]+1 .[11-\mathrm{b}, \mathrm{e}]$ 
equipped with a photomultiplier (Hamamatsu Photonics R 928 photomulitiplier tube), or to a spectral multichannel analyzer (SMA) system (Princeton Instruments, Inc. model TRY$700 \mathrm{G} / \mathrm{R} / \mathrm{Par}$ ). Films were prepared as follows. A 10 wt \% PMMA solution containing $0.01 \mathrm{~mol}$ $\mathrm{dm}^{-3}$ of CDB and $0-0.035 \mathrm{~mol} \mathrm{dm}^{-3}$ of TCT was made up in cyclohexanone. Films were $1.2 \mu \mathrm{m}$ in thickness prepared by spin-coating the solution onto a sapphire cell.

\section{Result and Discussion}

Laser flash photolysis in PMMA film. The maximal absorption for CDB were found at 780 and $790 \mathrm{~nm}$ in acetonitrile and a PMMA film respectively, as depicted in Fig. 2. The maximal fluorescence, $875 \mathrm{~nm}$ in a PMMA film with $355 \mathrm{~nm}$ exciation in Fig. 3.

On the excitation of a PMMA film containing CDB by $355 \mathrm{~nm}$ pulse, a weak fluorescence was observed, but any transient absorption was not observed. The fluorescence decay would be too short to be determined by 20 ns laser pulse.

Fig. 4 shows the quenching of fluorescence at various concentrations of TCT at an excitation of $355 \mathrm{~nm}$. The fluorescence intensity was decreased as the concentration of TCT changed from 0 to $0.32 \mathrm{~mol}$ $\mathrm{dm}^{-3}$.

As for quenching reduction of fluorescence in film, Kaneko et al. reported a multistep equilibrium model (Eqs. 1-12)[8]. In the equations, the multistep equilibrium between one CDB and more than two TCTs within a quenching sphere is assumed (Eqs. 1-2). $\mathrm{CDB}$ and (CDB-iTCT) is photoexcited to an excited singlet state 'CDB (Eq. 3) and '(CDB-iTCT) (Eq. 8) by absorbing a laser pulse. 'CDB undergoes i) radiative (Eq. 5) and nonradiative deactivation (Eq. 4), ii) intersystem crossing (Eq. 7), and iii) dynamically sensitized decomposition of TCT, forming radicals such as chloro and dichloromethyl radicals (Eq. 6). '(CDB-iTCT) undergoes i) radiative (Eq. 10) and nonradiative deactivation (Eq. 11), ii) intersystem crossing (Eq. 12), and iii) statically sensitized decomposition of TCT, forming radicals such as chloro and dichloromethyl radicals (Eq. 9). The attenuation of fluorescence by TCT in film is described as Eq. (13). When '(CDB-iTCT) does not emit fluorescence, $\mathrm{kq}^{\prime}$ is much larger than any other decay rate. Eq. 13 is described as a combination equation (Eq. 14) of dynamic and static quenching term (perrin type [9]).
The logarithmic plots of the fluorescence intensity at various concentrations of TCT was fitted to Perrin Equation. The quenching radius $\left(\mathrm{R}_{\mathrm{f}}\right)$ was $7.8 \AA$ for $\mathrm{CDB}$, which was determined from the slope of the logarithmic plots. The quenching radius is rather short compared to that obtained for photosensitization systems in which an exothermic electron transfer from photoexcited dyes to radical generators takes place; for examples, $R_{f}=15 \AA$ for 3-(9-julolidinyl)-1-

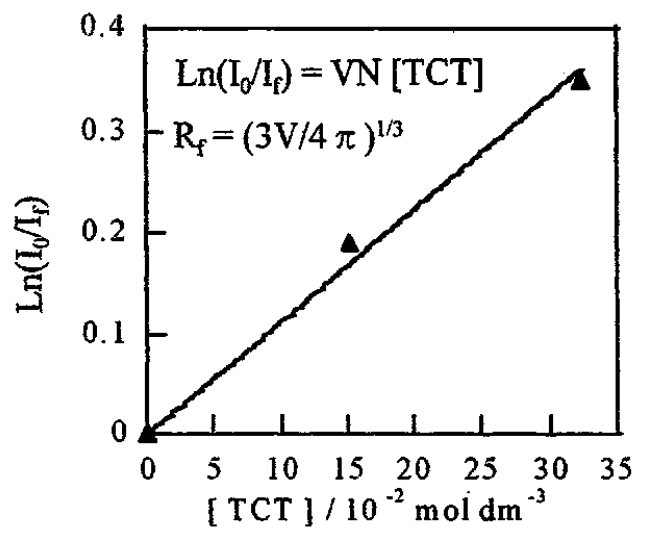

Fig. 4. Fluorescence Quenching of $\mathrm{CDB}$ by TCT in PMMA.

Substance concentration: $1 \times 10^{-1} \mathrm{~mol} \mathrm{dm}^{-3}$ for CDB; excitation wavelength: $355 \mathrm{~nm}$; detection wavwlength $890 \mathrm{~nm}$.

pnenyl-2-propen and TCT, and $R_{t}=14 \mathrm{~A}$ for 2-(9-julolidinyl)methylene-1-indanone and TCT [7-e]. The short quenching radius would be due to the low-singlet-excited energy of infrared dye, which made the energy of electron transfer less exothermic. It is concluded that the singlet static sensitization process is predominant in the photopolymer layer containing an infrared dye, $\mathrm{CDB}$, and TCT.

\section{References}

1. a) J. L. Seifert, R. E. Connor, S. A. Kushon, M. Wang, B. A. Armitage, J. Am. Chem. Soc., 121, 2987 (1999), b) B. K. Murnnally, H. He, L. C. Li, S. A. Tucker, L.B. McGown, 69, 2392 (1997).

2. A. Naber, U. C. Fischerand, S. Kirchner, T. Dziomba, G. Kollar, L. F. Chi, H. Fuchs, J. Phys. Chem. B. 103, 2709, (1999).

3. M. Kaschke, N. P. Emsting, B. Valeur, and J. Bourson, J. Phys. Chem. 94, 5757 (1990).

4. T. Urano, H. Nagasaka, M. Shimizu and T. Yamaokam. shimizu and T. Yamadka, J. Imag. 
Sci. and Technol., 41, 2,407 (1997).

5. A. Sanchez-Galvez, P. Hunt, M. A. Robb, M. Olivucci, T. Vreven, and H. B. Schlegel, J. Am. Chem. Soc., 122, 2911 (2000).

6. H. Masuhara, "Photophysical and Photochemical Tools in Polymer Science", ed M. A. Winnk, D. Reidel Pub. Comp., 1986, P. 65.

7. a) T. urano, H. Nagasaka, M. Tsuchiyama and S. Shimizu, Mitsubishi

Kasei $R$ and D., 2, 73 (1991), b) T. Urano, H. Nagasaka, M. Tsuchiyama, S. Shimizu, K. Kawazoe, M. Shimizu and T. Yamaoka, Bull. Chem. Soc. Jpn., 68, 1661 (1995), c) T. Urano, H. Nagasaka, M. Shimizu, S. Takahara and T. Yamaoka, Bull. Chem. Soc. Jpn., 69, 693 (1996), d) T. Urano, H. Nagasaka, S. Shimizu, H. Ito, M. Shimizu, S. Takahara and T. Yamaoka, Bull. Chem. Soc. Jpn., 70, 1659 (1997), e) H. Itoh, A. Takada, H. Kudo, H. Yokoyama, T. Urano, and H. Nagasaka, Bull. Chem. Soc. Jpn., 70, 2221(1997).
8. a) K. Nagai, N. Takamiya, and M. Kaneko, Macromolecule. Chem. Phys., 197, 2983 (1996), b) S. Ishikawa, T. Nishijima, T. Abe, K. Nagai, and M. Kaneko, J. Chem. Soc., Faraday Trans., 94, 73 (1998), c) K. Nagai, J. Tsukamoto, N. Takamiya, and M. Kaneko, J. Phys. Chem., 99, 6648 (1998), d) K. Nagai, N. Takamiya, and M. Kaneko, J. Inorg. and Organometal. Polym., 4, 391 (1994), e) K. Nagai, Y. Ueno, N. Takamiya, and M. Kaneko, Macromol. Chem. Phys., 196, 1241 (1995), f) K. Nagai, T. Nishijima, N. Takamiya,M. Tada, and $M$. Kaneko, $J$. Photochem. Photobiol. A: Chem., 92, 47 (1995), g) J. Zhang, F.Zhao, and M. Kaneko, $J$. Porphy. Phthalocyan., 4, 65 (2000).

9. J. B. Birks, Photophysics of Aromatic Moleculers, Wiley Interscience, London, P. 441 (1970). 